research, the anomalous pneumo-streptococcus, the repeated recovery of which has been a feature of the research, has, for convenience and following Ortner's terminology, been designated as the micrococcus pneumoniæ.

Of the 61 charts in the original manuscript only 30 have been selected for publication owing to considerations of space and the difficulty of reproducing such a large number.

\section{Reading of Charts.}

The recognition of certain salient points is a necessary preliminary to any attempt at reading the charts.

The common base line of every chart represents a temperature of $98.4 \circ \mathrm{F}$. and an opsonic index of 1.00 ; for convenience one degree of temperature corresponds to four divisions of the opsonic scale. The normal limits of the tuberculo-opsonic index are arbitrarily fixed as lying between 0.80 and $1 \cdot 20$. With the exception of the micrococcus catarrhalis it has been determined in the course of numerous experiments that the defined above wide limits are fully adequate in the case of the staphylococcus, most strains of the micrococcus pneumoniæ, the bacillus of Friedländer, the bacillus coli, the diplococcus pneumonix. The intracellular digestion of the micrococcus catarrhalis atter an incubation of only ten minutes renders accurate counting impossible, so that any but wide variations of the opsonic index should be rejected as being inconclusive.

The degree of variation of the opsonic curves is of paramount importance; while readings of the tuberculoopsonic index above 1.7 and below 0.4 are very uncommon, the range of variation of the other organisms is far greater. Further, whereas a temperature of $102^{\circ}$ would in the case of active tuberculosis call for serious comment the tuberculoopsonic index of $2 \cdot 1$ which corresponds with it on the opsonic scale is of far greater significance. It is essential to emphasise the above considerations.

Apart from the value of abnormal indices as evidence of infectivity certain contrasts manifest themselves to a greater or lesser degree in some of the charts. An attempt has been made to make this apparent in the arrangement of the charts. To consider this in more detail. An inverse relationship of the blood curve to the temperature is observed in ten charts. The blood curves are inverse in eight cases. In other charts this inverse relation cannot be traced, though in a series high indices to the tubercle bacillus are opposed to low indices to other organisms, or vice vers $\hat{a}$.

The inverse relation of the temperature to the elaboration of protective substances in acute bacterial infections is undisputed-e.g., acute lobar pneumonia, Mediterranean fever, typhoid fever, and streptococcal infections-and to a certain degree in acute tuberculosis. The recurrence of suggestive correlations between the temperature and blood curves in the protocols points to the complex rather than the simple origin of the fever in pulmonary tuberculosis.

The first cases presented are those belonging to Group I. i.e., "resting febrile" cases. They are subdivided as follows: (a) Infection by the tubercle bacillus and other organisms; $(b)$ infection by the tubercle bacillus alone; and (o) no evidence of infection by the tubercle bacillus, but infection by other organisms.

The temperature charts record the morning and evening temperature of each patient during the ten days preceding and following the day on which the blood examination was carried out. This day is indicated by a star in most cases. T, Temperature curve. R. To, Rectal temperature curve. $O$, Tuberculo-opsonic index curve. $P$, Curve of the opsonic index to the pneumo-streptococcus. S, Curve of the opsonic index to staphylococcus. C, Curve of the opsonic index to micrococcus catarrhalis. L, Curve of the number of leucocytes per c.mm. of the blood. The arrows indicate that the patients took walking exercise or worked for varying periods of time immediately before the next following temperature was recorded and the second sample of blood drawn.

\section{Table of Results.}

$$
\text { TABLE I. }
$$

Group 1, "Resting Febrle."

A. Infection by tubercle bacilli and secondary organisms ... 25

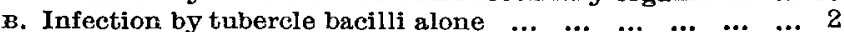

c. Infection by secondary organisms alone .......................

Group 2, "Ambulant Febrile, Resting Afebrile."

A. Infection by tubercle bacilli and secondary organisms ... 3

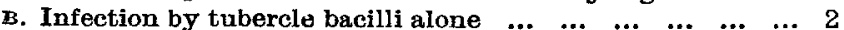

c. Infection by secondary organisms alone $\ldots .$.
Group 3, "A mbulant A febrile."

Cases.

A. Infection by tubercle bacilli and secondary organisms $\ldots 9$

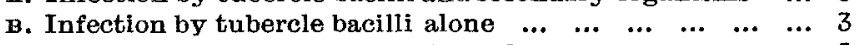

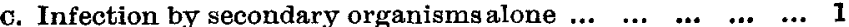

Group 4, "Working Afebrile."

A. Infection by tubercle bacilli and secondary organisms $\ldots 4$

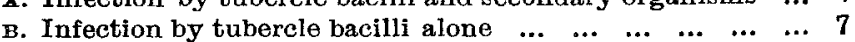

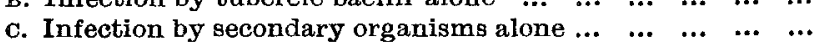

TABLE II.

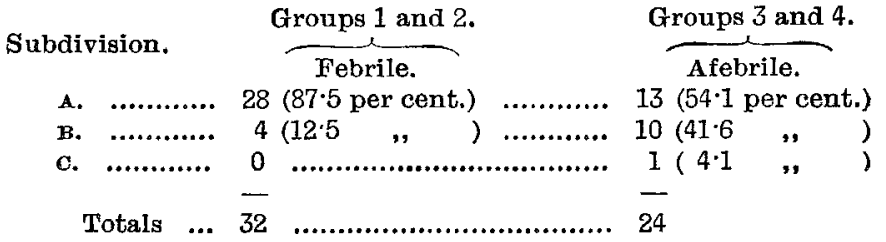

\section{Conoiusions.}

1. In nearly every case of open tuberculosis of the lungs the tubercle bacillus is the predominant infecting agent.

2. The blood examinations have shown that "secondary infections" do occur. In all cases "resting febrile" there was evidence of such infection. Even in the "ambulant afebrile " group such infection could not be excluded.

3. The temperature chart alone cannot determine the presence or absence of a secondary infection.

4. A consideration of the morbid anatomy of advanced tuberculosis, and of the uncontrolled auto-inoculations spontaneously occurring in such cases, precludes the hope of a successful issue from specific treatment directed against the secondary infections; the situation is more encouraging in afebrile cases.

It is a pleasure to record my indebtedness to the medical staff at the Brompton Hospital for generously placing their cases at my disposal, and to Dr. Paterson for selecting the Frimley patients for me. The work could not have been accomplished without the loyal coöperation and constant help of Dr. Cecil Clarke, assistant in the hospital laboratory.

\section{CORNEO-SCLERAL TREPHINING}

(LIEUTENANT-COLONEL R. H. ELLIOT'S OPERATION).

BY B. T. LANG, F.R.C.S. Eng., M.A., B.C. CANTAB., OLINICAL ASSISTANT TO THE ROYAL IONDON AND THE CENTRAL LONDON OPHTHALMIO HOSPITALS, AND TO THE OPHTHALMIO DEPARTMRNTS OF ST. BARTHOLOMEW'S AND MIDDLESEX HOSPITALS.

IT is widely admitted that every kind of successful operation for primary glancoma produces a leak and that within certain limits the success of the operation is proportional to the amount of leakage produced. In many operations the eventual leakage area is of uncertain size, and depends on the extent of the failure of scar formation in the wound. On this account the results obtained by such operations are far better in elderly people with feeble powers of repair than they are in middle-aged people whose wounds heal soundly.

Lieutenant-Colonel R. H. Elliot, I.M.S., has solved the problem. From beneath a conjunctival flap he removes a large portion of the corneo-sclerotic with a trephine. The resulting hole is too wide to become occluded by scar tissue. But in order to obtain efficient leakage the hole must be made into the anterior chamber. The aqueous can then escape into the spaces of the subconjunctival tissue.

So far as I am aware, all the cases of failure of this operation have resulted from one of two causes-either because the trephine hole is too small and gets blocked by scar tissue, or because it is made ton far back (Fig. 5) and gets blocked sooner or later more or less completely by the iris or iridic exudate.

The description of the operation may conveniently be divided into preliminary considerations and three stagesnamely: (1) The reflection of the conjunctivo-corneal flap; (2) the act of trephining; and (3) the treatment of the trephined disc and of the iris.

$$
\text { Preliminary Considerations. }
$$

Choice of site of operation.-It is usual to make the trephine hole above, so that the upper lid may cover and protect it; above and below the limbus extends further on to 
the cornea, and therefore in these situations it is easier to get well into the anterior chamber. If the operation is performed at the side of the cornea care must be taken that the conjunctivo-corneal flap is carried far enough forward.

Size and shape of the fap. - It is essential that the flap be made large, so that slight displacement will not leave the hole uncovered. So long as this condition is fulfilled the shape of the flap is unimportant.

Stage 1. -The Reflection of the Conjunotivo-corneal Flap.

The eye is cocainised, the usual toilet of the conjunctiva performed, and a speculum introduced. The patient is asked to look down, and the conjunctiva is seized with a pair of conjunctival forceps at a point about $8 \mathrm{~mm}$. from the corneoscleral junction directly above the centre of the cornea. This fold of conjunctiva, the axis of which is a continuation of a radius of the cornea, is cut through with scissors at that end which is distal to the corneal margin. The flap is roughly triangular in shape, and is made by continuing this incision in both directions in a suitably curved manner. (Fig. 1.)

FIG. 1.

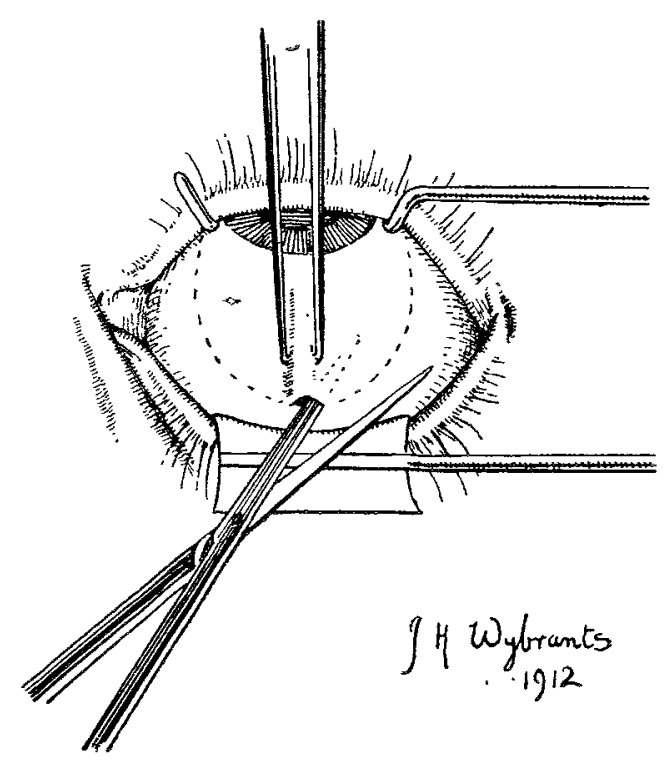

Right eye from above and behind as seen by the surgeon during the first stage of the operation.

The incision should end on either side at points about 3 to $5 \mathrm{~mm}$. from the corneal margin at a level with the junction of the upper and next quarter of the cornea. This part of the operation is most easily carried out by opening the blades of the scissors, slipping one blade under the conjunctiva and closing them, as shown in the diagram. This is a far better method than taking small snips with the points of the scissors. The flap is then dissected off the underlying sclera by snipping boldly with sharp pointed scissors which are held flat against the surface of the sclera. If the flap is held with forceps it is easily torn. It is better to draw it gently towards the centre of the cornea, using small pieces of folded lint or pledgets of cotton-wool held in forceps. These swabs can be used to absorb any blood that exudes from the cut vessels. Bleeding is usually slight, but in cases of acute glaucoma or in cases where the conjunctival vessels have been recently engorged by the use of perchloride or other irritant lotion there may be considerable bæmorrhage.

In the normal eye it is quite easy to separate the conjunctiva from the sclera right up to the limbus, but in cases which are the subjects of chronic glaucoma the conjunctiva

FIG. 2.

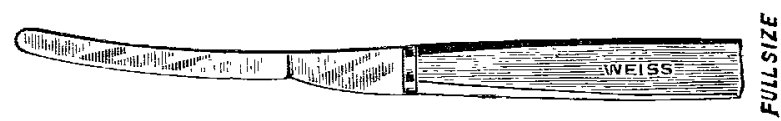

Desmarres' secondary cataract knife.

is bound down to the underlying tissue for as much as 3 to $4 \mathrm{~mm}$. from the corneal margin. Under these circum. stances the reflection of the flap requires care and patience to avoid button-holing. The flap can no longer be raised easily by snipping. The fine fibrous bands binding the two together are best divided by scratching or cutting. For this purpose the point of one blade of the scissors may be used. But this part of the operation is greatly simplified if the surgeon uses a Desmarres' secondary cataract knife which is modified by having the whole of the blunt end sharpened (Fig. 2). With the aid of this instrument the reflection of the flap is much less difficult, and takes about a quarter of the time. There is another advantage ; in all operations depending for their success on leakage it is as desirable to cause as little damage as possible at the site of operation, because damage leads to inflammation, and inflammation leads to an excessive formation of scar tissue The clean cut of a knife is much less likely to set up inflammation than is the scratching with the point of the scissors, and is therefore to be preferred on these grounds alone.

The sub-conjunctival tissue in the neighbourhood of the limbus is denser than elsewhere; when this region is approached the base of the retracted flap has a roll-like appearance which has been compared to the corona glandis penis. The reflection of the flap is continued until a small portion of the superficial layer of the cornea has been reflected. (See Fig. 4.)

Directly the white opaque fibres of the limbus (or sclera) have been divided, or directly the dissection has been carried beyond them, the light passes through the underlying translucent cornea and the bottom of the wound appears black. This is a landmark of great importance, and until it is seen the flap has not been carried far enough forward. The cornea should be split up for from $\frac{1}{2}$ to $1 \mathrm{~mm}$. When the central part of the base of the flap has been reflected far enough attention should be paid to the edges. These should be reflected back to about the same level. The site for trephining will then be well exposed and not half covered by a hood of conjunctiva. This is important as it is very difficult to trephine at the bottom of a pocket.

$$
\text { Stage 2.-The Aot of Trephining. }
$$

The trephine should be 1.5 or $2 \mathrm{~mm}$. in diameter. It should be quite sharp, so that the least possible damage may be done to the edges of the hole. The trephine and holder should be light in weight so that the least alteration in resistance may be easily appreciated. The holder should be so shaped that it can be rotated between the fingers with facility, and should have a shoulder to prevent the fingers slipping down on to the blade during operation. It is further advisable to have some means of preventing the blade being damaged during sterilisation. In the pattern illustrated, which has been made by Messrs. Weiss, the blade can be drawn within the handle and clamped in that position. (Fig. 3)

$$
\text { FIG. } 3 .
$$
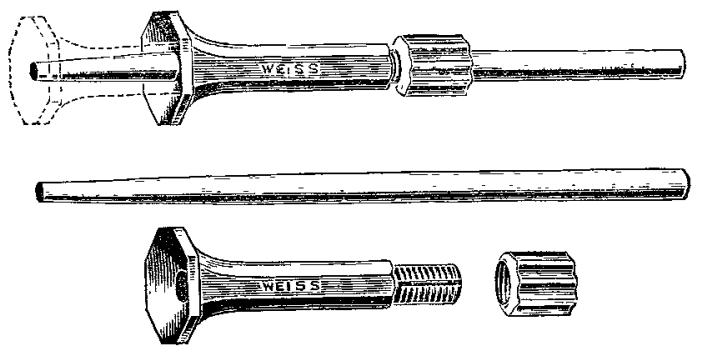

The solid part of the upper figure is the instrument ready for use. The blade can be pushed out to suit the operator. The skeleton outline represents the blade drawn into the handle for protection during boiling. The middle figure is the steel tube, and the two lower figures are the handle and its nut, which clamps the blade and holds it firm.

When the conjunctival flap has been reflected the trephine is placed on the prepared area as far forward as it is possible without button-holing the flap. Small rotatory movements are given to the trephine by rolling it backwards and forwards between the thumb and finger (cigarette rolling motion). Only the slightest possible amount of pressure should be applied. Shortly after the trephine has started cutting it is advisable to remove it and to inspect the cut to see that it is in the right place. If it is found that the trephine has slipped a new hole should be started. Great care should be taken to keep the axis of the trephine perpendicular to the area of contact as it is highly desirable that one side of the hole should not be cut through much before the other.

When the trephine has cut through the corneo-sclerotic 
there is a feeling as if the end of the instrument were being sucked into the wound. This is only felt if one is using a light trephine and if the trephine is so sharp that no pressure has to be used to make it cut. As it is a point of great importance to know when one is through, it is a point of equal importance that the trephine be light and sharp.

The trephine is now removed. If the disc has not been cut free all round the experienced operator may replace the trephine and cut through the tissue retaining the disc. It is a great deal safer to deal with the disc with forceps and scissors, as described below. The lens may easily be injured by persevering too long with a blunt or heavy trephine. (Fig. 4.)

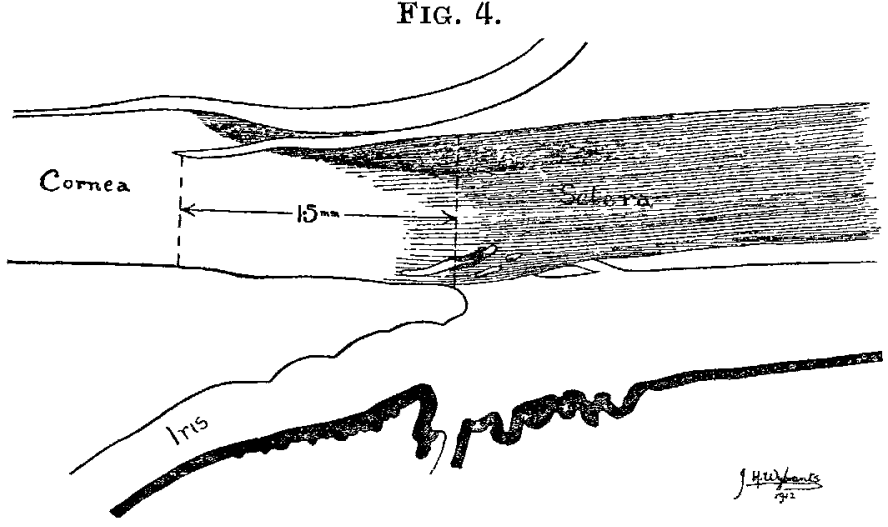

Drawn from a section of a normal eye. The eye was excised during an operation for sarcoma of the upper jaw and orbit. It was fixed in 5 per cent. formalin in normal saline. A corneo-conjunctival flap was reflected from above as described in this paper. Portions of the eye were cut away on a freezing microtome until the section passed vertically through the centre of the cornea. With the aid of a small amount of gum a slide was frozen on to the cut surface. The eye was now thawed off the microtome, and the slide with the eye still frozen to it was reversed, placed on the microtome, and with the aid of a little gum frozen on to it. The eye was then cut away once more until a very thin layer was left. A cover-slip was laid on and the whole was allowed to thaw. This procedure was adopted to avoid any of the shrinkage effects associated with the usual methods of embedding tissues for section. This particular section varied in thickness in different parts, from 12 to $28 \mu$. The drawing was made from the section. The dotted lines show the amount of the corneo-sclera removed by a $1.5 \mathrm{~mm}$. trephine. It will be-seen that the splitting of the cornea enables one to make the trephine hole so far forward that it is impossible for it ever to become blocked by iris or iridic exudate. Should be compared with Fig. 5 .

Stage 3.- The Treatment of the Trephined Diso and of the Iris.

When the disc has been cut through one of three things may happen to both the iris and the disc: (1) The iris may prolapse through the wound; (2) it may bulge into the wound ; or (3) it may remain in its normal position. If the iris prolapses or bulges it forces the trephined disc completely or partially out of the wound; while if it remains in its normal position the disc may stay in the hole, or it may be sucked into the anterior chamber.

The disc has to be removed and the iris, if prolapsed or bulging, has to be dealt with. The disc may be removed by seizing it with iris forceps and cutting it free in the wound from its attachment to the sclera with a pair of fine sharppointed scissors. The object is to complete the trephine cut if possible. If the disc is merely drawn out of the hole and as much as protrudes cut off, it is quite possible that a flat section of the disc may be left at the bottom of the hole so as to occlnde it completely. This is particularly likely to occur if the trephine hole has been made too far back, where the under surface of the disc will be adherent to the base of the iris.

The bulging iris may present a slight difficulty during this stage; it may be convenient to snip the most prominent part with a pair of scissors and allow the aqueous to escape. The iris will then subside and the disc can be dealt with as above described.

If the disc has receded into the anterior chamber it can generally be washed out by the use of an irrigator. In these cases the disc is usually adherent to the edge of the hole, and it comes back into the hole with a hinge-like movement. It may be easily seized with forceps and removed, observing the precautions already mentioned.

With regard to the iris, if it does not readily return as much as remains outside the eye should be removed with scissors. If it returns but the pupil does not become central the cornea should be gently stroked with the back of a curette or an iris repositor starting from the side of the cornea opposite the hole and stroking towards the hole. The cornea is very likely to be dry and should first be moistened. If the pupil still remains drawn up it is best to introduce Liebreich's or other iris forceps through the hole, draw out some of the iris, and do a small iridectomy. The edges of the iridectomy will easily be freed by stroking the cornea as above described. If the hole has been made too far back an iridectomy must be performed, as otherwise the operation is almost bound to fail. (Fig. 5.)

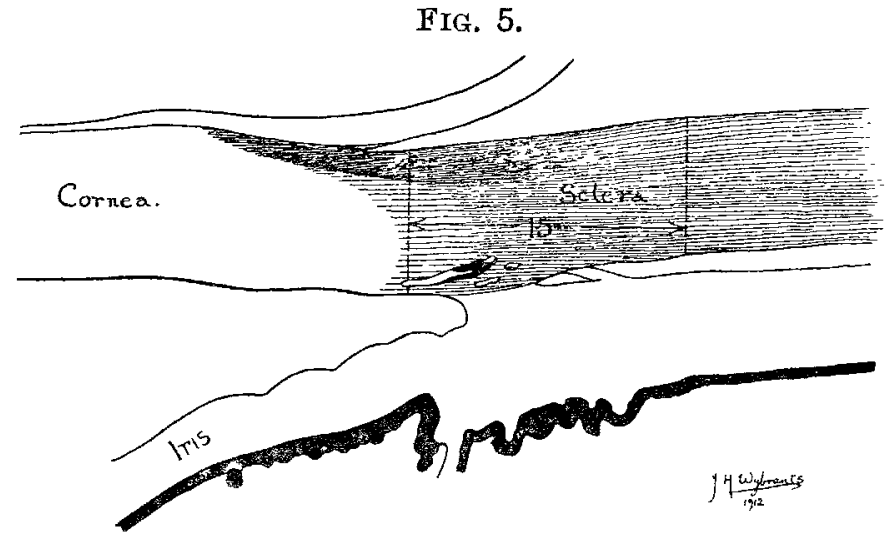

A diagram made from Fig. 4 to show where the trephine hole would come if the flap is insufficiently reflected. Fyes that have been operated upon in this manner are relieved for a time, but later the tension again rises. In such eyes a bridge of white tissue of the limbus or sclera is seen to intervene between the trephine hole and the cornea.

The conjunctival flap is now drawn into its original position and the upper lid lifted over it as the speculum is removed. If the flap has been made from below it is better to put in one stitch, as flaps in this position are easily displaced. A pad and bandage are applied.

In this short consideration of a comparatively new operation it has not been thought advisable to multiply unnecessary detail, particularly as those practising it will have already had considerable experience in the ordinary technique of ophthalmic operations.

I am greatly indebted to Lieutenant-Colonel Elliot for teaching me the technique of this most important operation. No other operation for glaucoma is so certain of success. The wound in the corneo-sclera is so small that the contents of the globe cannot be extruded, and the restless patient is less able to do permanent damage to the sight by screwing up his lids or hitting his eye. Provided that the trephine hole is made well into the anterior chamber and the wound is freed from the trephined disc and displaced iris, the success of the operation is assured.

I should like to take this opportunity of thanking SurgeonGeneral P. H. Benson, I.M.S. (retired), for so kindly permitting me to work under Lieutenant-Colonel Elliot at the Government Ophthalmic Hospital, Madras.

I append a list of publications by Lieutenant-Colonel Elliot on trephining: 1. Preliminary Note on a New Operative Procedure for the Establishment of a Filtering Cicatrix in the Treatment of Glaucoma, Ophthalmosoope, December, 1909. 2. The Operative Treatment of Glaucoma, ibid., July, 1910. 3. Trephining for Glaucoma, ibid., November, 1910 . 4. The Operation of Trephining for Glaucoma, its History, its Technique, its Indications, and its Results (an address delivered at the Oxford Ophthalmological Congress), ibid, July, 1911. 5. Simple Trephining for the Relief of Glaucomatous Conditions (a paper read berore the Section of Ophthalmology at the seventy-ninth annnal meeting of the British Medical Association in Birmingham, July, 1911). 6. The Operation of Simple Trephining of the Sclera for the Relief of Glaucoma, Ophthalmology, April, 1911. 7. A Modified Instrument for the Performance of Simple Trephining for the Relief of Glaucoma, Ophthalmosoope, January, 1912. 8. Some Technical Details of the Operation of Simple Trephining, Ophthalmological Society's Transactions, Vol. XXXI., June 8th, 1911

Cavendish-square, $\mathbf{W}$. 